\title{
CONOCIMIENTOS Y AUTOEFICACIA PARA LA APLICACIÓN DEL PAE EN ESTUDIANTES DE ENFERMERÍA
}

Mira. Martha Liha Bernal Beccerni". Mitra Gandhy Ponce Gómez. * y Mitra Liliana Gonzalez Juárez...*

"Protesar de Carrera Asociado B". De TC de la ENEO, "* Profesor de Carrero Asociado "B". De T.C de la ENEO "* Pratesor de Carrera Titular "A" de la ENEO

\section{RESUMEN.}

La Enfermería, tiene como práctica social los cuidados ante situaciones de salud, siendo el Proceso Atención de Enfermería (PAE) el método de intervención que la distingue y resulta indispensable en los ámbitos de enseñanza y de práctica, además de profundizar en el conocimiento del cuidado y su aplicación práctica.

Estudio cuantitativo - correlacional en una población de estudiantes de Enfermeria de licenciatura ( $N=292)$, para reconocer diferencias entre estudiantes de programas intermedios y avanzados, en las dimensiones de conocimientos y autoeficacia para aplicar el PAE.

Los estudiantes de semestres avanzados poseen más conocimientos y nivel de autoeficacia, siendo los factores influyentes más sobresalientes las experiencias de contacto con el cuidado, edad, y el considerar el modelo de mayor credibilidad al docente.

La adquisición de conocimientos es indispensable para el desarrollo personal y profesional del individuo incluyendo en ello su capacidad de comprensión de la realidad y de actuación sobre ella, lo que implica relaciones interpersonales donde educando y educador aprenden de la asimilación y acomodación de esa información.

La autoeficacia desempeña un papel fundamental en el funcionamiento humano, influyendo el comportamiento por su impacto ante metas y aspiraciones, asi como los impedimentos y oportunidades que se le presentan en el medio social.

Con el fin de fortalecer la enseñanza del cuidado y las habilidades de la práctica de Enfermería es necesario fortalecer los mecanismos de autoeficacia tanto como los conocimientos y fortalecer el cuidado profesional para la salud de los individuos.

PALABRAS CLAVE: Conocimientos, Autoeficacia, Proceso Atención de Enfermería.

\section{ABSTRACT.}

Nursing has, as its social practice care facing health situations, being the Nursing Attention Process (PAE) the intervention method that distinguishes attention. It is indispensable within teaching and practice scopes; as well as getting deep into caring knowledge and its practical application.

A quantitative-correlated study on a nursing career students population ( $N=292)$ in order to recognize differences among intermediate and advanced program nursing students, in knowledge and self-efficiency dimensions for applying the PAE.

Advanced semesters students have more knowledge and a higher self-efficiency level, being the more outstanding influence factors the contact experiences with care, the age, and considering the model with major credibility to the teacher.

The knowledge acquisition is indispensable to personal and professional development of individual including its reality and performance capacity of comprehension about it, that implicate interpersonal acquaintances from this assimilation and put in order that information.

Self-efficiency plays a fundamental roll in human functioning, influencing behavior due its impact facing goals and aspirations, as well as because the hindrances and opportunities it has to face within the social scope.

Aiming to strengthen care teaching and the nursing practical abilities it is necessary to make stronger the selfefficiency mechanisms as well as knowledge, and strengthen professional care for the individuals health.

KEY WORDS: Knowledge, Self-efficiency, Nursing Attention Process. 


\section{INTRODUCCIÓN}

La Escuela Nacional de Enfermeria y Obstetricia tiene entre sus tareas la formación de "profesionales de Enfermería competentes, informados y con conciencia de la responsabilidad social de cuidar a las personas, grupos y comunidades en los procesos de salud y enfermedad en sus ambientes cotidianos o en el entorno donde acuden a atender su salud, siendo el eje de esta experiencia educativa el cuidado: y teniendo como herramienta metodológica el proceso de Enfermeria para implementar su intervención profesional"

El docente Enfermero es el responsable de propiciar el análisis y reflexión del Proceso de Atención de Enfermería (PAE) para su uso y aplicación en la práctica diaria, que tenga por consecuencia un incremento en la calidad de la atención y el fortalecimiento profesional asi como el de la disciplina. Es asi que el futuro egreso de estudiantes de la carrera, los coloca como aplicadores potenciales de esta herramienta metodológica.

Una forma de profundizar en la metodología del cuidado, es reconocer la importancia del nivel de conocimientos para la implementación del proceso de Enfermería en el ámbito clinico, asi como identificar la percepción de autoeficacia que se posee para aplicar dichos conocimientos ante una situación real, además este método es el sustento de la práctica en la disciplina, que se encuentra considerado en el Plan de Estudios de la Licenciatura en Enfermeria y Obstetricia. (LEO).

Como antecedente existe un estudio de Franco y Benítez (2000) quienes reportaron que el PAE en la práctica clínica solo se lleva a cabo ocasionalmente por resultar un proceso complejo, tedioso y dificil de aplicarlo a la actividades diarias de Enfermería

De acuerdo con la teoría cognitiva social, la autoeficacia es la percepción de la habilidad y la competencia en un. dominio particular, que motiva a los individuos a realizar

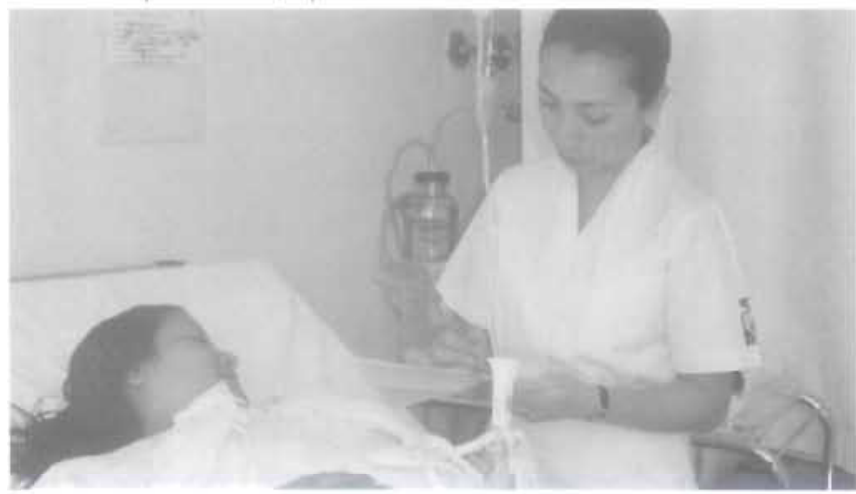

aquellas conductas en las que sobresalen. Asi la percepción acumulativa de eficiencia, determina la predisposición para realizar una conducta dada? donde dicho dominio se origina a partir de los conocimientos, la experiencia - las habilidades prácticas propias de la profesión?.

Bandura establece que para que los individuos puedan hacer bien las cosas, requieren poseer dominio en el área que manejan'; reconoce a la autoeficacia como influyente en el logro de determinados resultados, planteando mecanismos fundamentales para lograrla a través de: trabajar sobre éxitos, modelaje (vicario) y persuasión verbal.

a) Trabajar sobre éxitos: implica situaciones donde un individuo o grupo realiza conductas sencillas en las que se garantice el éxito, donde el aumento paulatino de la complejidad de las tareas, hace que la autoeficacia vaya incrementando y a su vez el profesional se encarga de hacer conciente a la persona de sus logros. Es decir, a través de la promulgación exitosa repetida de tareas incrementales, la persona adquiere anticipaciones mejoradas de éxito en una tarea dada, lo que a su vez afecta positivamente la persistencia, iniciación y duración de la tarea y promueve así el cambio de comportamiento.

b) Experiencia vicaria o modelaje: consiste en aprender por medio de escuchar u observar de las acciones o desempeño de modelos que pueden ser personas o símbolos no humanos; sin ejecución abierta del que aprende. Cuando una persona ve a alguien de condiciones parecidas a las que él mismo tiene, y que ha sido capaz de hacer aquello que éste se esta planteando hacer, aumenta la confianza en sí mismo.

Esta estrategia para incrementar la autoeficacia en Enfermería es factible de llevar a cabo con la intención de reforzar aprendizajes en los estudiantes.

c) Persuasión verbal: implica hacer conciente verbalmente al sujeto de la etapa en la que se encuentra de sus logros o retrocesos. A este respecto es que considero que si se trabaja constantemente con los alumnos haciéndoles notar los avances y errores en la aplicación del PAE en situaciones reales se esperaría que una vez haciendo conciente sus alcances y limitaciones reflexione sobre ellas y trate de superarlas.

El P.A.E. como un proceso reflexivo y de intervención de Enfermería visto desde una postura cognoscitiva, no es un proceso aislado o solo controlado por fuerzas 
internas, sino que se ha descubierto que los eventos intelectuales son moldeados automáticamente por estímulos externos, lo que incluye la parte instrumental del método.

\section{METODOLOGÍA}

Se trata de un estudio, correlacional-transversal entre alumnos de $4^{\circ}$ y $8^{\circ}$ semestre de una escuela de Enfermería de nivel superior en el D.F. donde la $N=250$ alumnos.

Los datos fueron procesados en el paquete estadístico SPSS V.1 1.0. Se realizó un análisis descriptivo y un análisis inferencial con problemas de comparación y relación por medio de las pruebas t de student y de r de Pearson.

El instrumento para valorar conocimientos es una adaptación del instrumento "Factores que Intervienen en el Conocimiento y Aplicación del Proceso de Cuidado" (FICAPC) en su apartado III, referente a conocimientos de proceso de cuidado ${ }^{6}$. Su escala varió de 0 a 10, siendo 0 el minimo que significa que "no identifica el concepto de PAE, etapas ni su uso", mientras que el 10 o máximo nivel, significa que "se identifica clara y ampliamente el concepto y etapas del PAE" (Ver cuadro No. 1).

La variable autoeficacia es medida a través de la escala de Autoeficacia de Bandura en un instrumento adaptado para el estudio, que va de 0 a 100 donde 0 es "no puedo hacerlo" y 100 es "completamente seguro de poder hacerlo, a través de una escala tipo Likert (Cuadro No. 2). La sección de autoeficacia alcanzó un alpha de Cronbach de .8736 ; el instrumento incluye además preguntas para valorar trabajar sobre éxitos, modelaje vicario y persuasión verbal, con la misma escala de medición.

\section{RESULTADOS}

El nivel de conocimientos exhibido por la población de estudiantes independientemente del semestre, tiende a ser elevada al mostrar una media de 4.06; lo que quiere decir que los estudiantes "identifican en forma amplia y claramente el Proceso de Atención de Enfermería y sus etapas".

El índice general de autoeficacia. mostró que el 56\% de la población está "completamente seguro" de aplicar el PAE (Media $=4.65$, esto quiere decir "seguro a completamente, seguro de poder aplicarlo").

Respecto a las dimensiones de Modelaje Vicario y Persuasión verbal se obtuvo que el $78 \%$ y el $71 \%$ de la población "siempre hayan tenido experiencias en la prác- tica clinica o comunitaria" relativas a estas dimensiones que coadyuvan a la autoeficacia; las cuales se confirman al observar medias de 4.66 y 4.46 para cada una respectivamente.

Se intentó conocer si existian diferencias en semestres intermedios y avanzados detectándose que los estudian= tes de $8^{\circ}$ semestre en el "indice de autoeficacia generalizada", "facilidad para aplicar el PAE", "planificación" y "ejecución" se perciben "completamente seguros de poder hacerlo"; mientras que la población de $4^{\circ}$ semestre en la etapa de "valoración" y "evaluación", asi como la dimensión de "modelaje vicario" y "persuasión verbal" consideran estar "completamente seguros de poder hacerlo" (Ver cuadro No. 3).

En el caso de nivel de conocimientos también se detectaron diferencias estadisticamente significativas a través de la t de student (prob. $0.000, t=13.810, g \mid=249$ ) lo que quiere decir que los alumnos de $4^{\circ}$ semestre "identifican relativamente el PAE", mientras que los de $8^{\circ}$ semestre lo hacen desde "suficiente hasta amplia y claramente" (Media 4.27) (Ver cuadro 3).

Respecto a la edad, se encontró que los estudiantes de 20 años o menos son quienes perciben estar "completamente seguros en su autoeficacia" para la etapa de planificación; al igual que los alumnos de 26 a 30 años para la autoeficacia generalizada y el nivel de conocimientos; y es el grupo de 31 años o más quienes reportan mayores indices en la dimensiones de facilidad para la aplicación, valoración, ejecución, evaluaciön, modelaje vicario y persuasión verbal. Esto muestra que son los alumnos de mayor edad son quienes se perciben más autoeficaces.

En la relación entre la variable autoeficacia y la facilidad para aplicar el PAE $\left(r_{\mathrm{p}}=+.654\right.$ y prob. $\left.=.000\right)$ se obtuvo una moderada fuerza directamente proporcional lo que quiere decir que los estudiantes que consideran que les es más fácil aplicar el PAE, es también más alto su nivel de autoeficacia.

\section{DISCUSIÓN.}

En este estudio se trató de establecer diferencias entre alumnos de semestres intermedios y de semestres avanzädos acerca de conocimientos y percepción de autueficacia para la aplicación del PAE, de tal forma que se pudieran establecer relaciones entre el saber teórico y el saber práctico, y distinguir si el saber teórico se incrementa con el tiempo al igual que la percepción de autoeficacia; ambos conforme aumenta la expertés o el nivel académico. 
El aprendizaje es un elemento complejo en el que se pone en juego el proceso cognitivo del alumno, a partir de experiencias de práctica profesional, por medio de la enseñanza maestro-alumno o por el deseo personal de conocer más. El proceso enseñanza aprendizaje se convierte en un medio para lograr que los individuos adquieran habilidades o destrezas mentales y prácticas a través de la interacción socioeducativa.

Un estudiante con alta autoeficacia se compromete más con las actividades que se le encomiendan y muestrạ un mayor involucramiento y persistencia, a pesar de las dificultades que se puedan encontrar.

El proceso por el cual el estudiante adquiere su autoeficacia es sencillo y bastante intuitivo: el estudiante se involucra en la realización de determinadas conductas (tareas), interpreta los resultados de las mismas, y utiliza esas interpretaciones para desarrollar sus creencias acerca de su capacidad para involucrarse en tareas semejantes en algún momento futuro, y actúa de acuerdo con las creencias formadas previamente. Canto menciona que, en la medida que los académicos fomenten las creencias de. autoeficacia, el rendimiento del alumno se incrementạá.

Es importante hacer notar que el nivel de autoeficacia percibida es mayor entre alumnos de semestres avanza- dos que en semestres intermedios, tal como lo señali Laschinger quién examinó la autoeficacia de estudiante: de Enfermeria de pregrado para llevar a cabo actividade: de promoción a la salud con clientes, encontrando que las percepciones de confianza aumentaron al avanzar sı programa educativo. Por lo tanto se coincide con este autor, en que al aumentar el nivel de expertés y gradc académico, se incrementa la autoeficacia percibida pari la realización de determinadas tareas.

Respecto a los modelos de quienes reciben más apoy los estudiantes, se detectó que son los docente Enfermeros quienes coadyuvan tanto a las aportacione teóricas como de aplicabilidad del P.A.E. en la práctica de cuidado, cuestión que coincide con Tresolini y Stritter quienes encontraron que la observación de modelos cre. íbles en combinación con práctica y ánimo reales po parte de los practicantes, influenció positivamente lo niveles de autoeficacia para asesoría en promoción d. salud de estudiantes médicos.

\section{CONCLUSIONES.}

El cuidado a la salud es la esencia de Enfermería, y es $\epsilon$ P.A.E. la herramienta metodológica recomendada emplear en el dominio de su práctica; en la medida qu el estudiante se involucre en la cultura del cuidado y'e

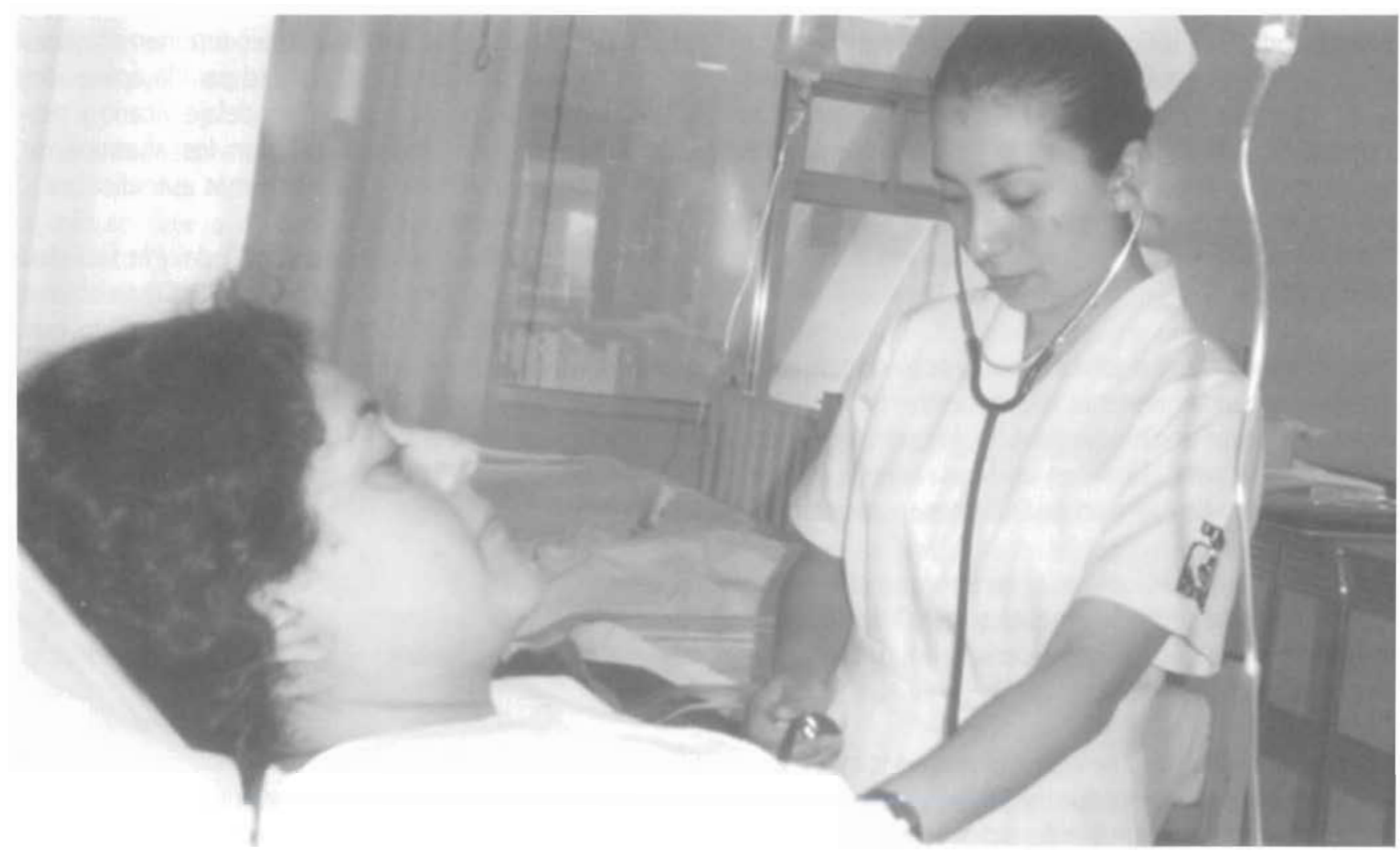


el uso del P.A.E. se va a lograr un mayor beneficio en la formación de éste como persona y como profesional.

La autoeficacia puede ser una brecha en el dominio de Enfermeria para generar apegos a la ejecución de actividades que se establezcan en el individuo para "el cuidado ".

Se coincide con Bandura en que los seres humanos adquieren destrezas y conductas de modo operante e instrumental donde intervienen factores cognitivos que ayudan al sujeto a decidir si adopta una determinada conducta o no. En el caso del estudiante de Enfermería se pretende que mientras más se domine el factor conocimiento vinculado a la adquisición de habilidades para la práctica o implementación del PAE; se logre el incremento de la autoeficacia y por lo tanto, el uso de esta metodología.

La autoeficacia tiene importantes implicaciones en el rendimiento académico del estudiante por lo que Canto? sugiere que los esfuerzos de los maestros deberían dirigirse a la motivación de las creencias de autoeficacia de los estudiantes acerca de su propia competencia como estudiante y como futuro profesional y que, para lograrlo, es necesario que los maestros diseñen estrategias de aprendizaje que permitan a los estudiantes adquirir confianza y competencia para realizar actividades que son propias para la adquisición del aprendizaje, en las se incluya el modelaje vicario, la persuasión verbal y el trabajar sobre éxitos.
De acuerdo con la teoria del Aprendizaje Social las experiencias vicarias (el aprendizaje por observación, modelamiento o imitaciọn) influyen en las expectativas de autoeficacia del estudiante cuando éste observa la conducta de otros estudiantes, ve lo que son capaces de hacer, nota lạs consecuencias de su conducta, y luego usa esta información para formar sus propias expectativas acerca de su propia conducta y sus consecuencias. La fuerza de las experiencias vicarias depende de algunos factores, según Canto influyen la semejanza entre el estudiante que observa y su modelo, el número y la variedad de modelos a la que se ve expuesto, la percepción de poder de ese modelo, y la similitud entre los problemas que afrontan el observador y el modelo.

La tercera fuente de autoeficacia se refiere a la persuasión verbal, es decir, el estudiante crea y desarrolla su autoeficacia como resultado de lo que dicen sus maestros, padres y condiscipulos. La retroalimentación positiva ("tú puedes hacerlo", "sé que lo lograrás") por parte de ellos puede aumentar la autoeficacia del estudiante, pero este incremento puede ser sólo temporal, si los esfuerzos que realiza el estudiante para realizar sus tareas no concluyen en el éxito esperado. La persuasión verbal influye menos en la creación y desarrollo de autoeficacia que el modelamiento vicario y el trabajar sobre éxitos incluso se asegura que la efectividad de la persuasión verbal depende de qué tan experta, confiable y atractiva sea percibida la persona que trata de persuadir.

Cuadro No. 1

Escala de Autoeficacia para aplicar el PAE.

\begin{tabular}{|c|c|c|c|c|}
\hline 120 & 40 & 60 & $61 \quad 80$ & $81 \quad 100$ \\
\hline No puedo hacerlo & $\begin{array}{l}\text { Apenas cierto de } \\
\text { poder hacerlo }\end{array}$ & $\begin{array}{l}\text { Relativamente seguro } \\
\text { de poder hacerlo }\end{array}$ & $\begin{array}{l}\text { Seguro de poder } \\
\text { hacerlo }\end{array}$ & $\begin{array}{l}\text { Completamente } \\
\text { seguro de poder } \\
\text { hacerlo }\end{array}$ \\
\hline
\end{tabular}

Fuente: Escala General de Autoeficacia de Albert Bandura, 1977.

Cuadro No. 2

Nivel de Conocimientos acerca de PAE

\begin{tabular}{|c|c|c|c|c|}
\hline $1.0 \quad 2.0$ & 2.1 & 6.0 & 8.0 & 8.1 \\
\hline $\begin{array}{l}\text { No identifica el } \\
\text { concepto y sus } \\
\text { etapas }\end{array}$ & $\begin{array}{l}\text { No identifica clara.- } \\
\text { mente el concepto } \\
\text { sus etapas }\end{array}$ & $\begin{array}{l}\text { Identifica relativa- } \\
\text { mente el concepto y } \\
\text { sus etapas }\end{array}$ & $\begin{array}{l}\text { Identifica suficiente- } \\
\text { mente el concepto y } \\
\text { sus etapas. }\end{array}$ & $\begin{array}{l}\text { Identifica ampliamen- } \\
\text { te el concepto y sus } \\
\text { etapas }\end{array}$ \\
\hline
\end{tabular}

Fuente: Benitez y Franco, adaptado por Bernal 2002. 
Cuadro No. 3

Comparación de dimensiones de Autoeficacia por Semestre

Dimensión

\begin{tabular}{|c|c|c|c|c|c|c|}
\hline & \multicolumn{3}{|c|}{40} & \multicolumn{3}{|c|}{80.} \\
\hline & Media & $\mathrm{N}$ & S.D. & Media & $\mathrm{N}$ & S.D. \\
\hline Autoeficacia & 4.63 & 140 & .485 & 4.68 & 110 & .523 \\
\hline Facilidad & 4.27 & 140 & .757 & 4.33 & 110 & .756 \\
\hline Valoración & 4.59 & 140 & .548 & 4.58 & 110 & .612 \\
\hline Planificación & 4.45 & 140 & .604 & 4.60 & 110 & .545 \\
\hline Ejecución & 4.41 & 140 & .668 & 4.59 & 110 & .579 \\
\hline Evaluación & 4.66 & 140 & .489 & 4.65 & 110 & .568 \\
\hline Modelaje vicario & 4.68 & 140 & .777 & 4.64 & 110 & .763 \\
\hline Persuación verbal & 4.49 & 140 & .835 & 4.42 & 110 & .839 \\
\hline Conocimientos & 3.90 & 140 & .733 & 4.27 & 110 & .703 \\
\hline
\end{tabular}

1. Escuela Nacional de Enfermeria y Obstetricia. Plon de Estudios de la Licenciatura en Enfermeria y Obstetricia. Ed. ENEO - UNAM. México, 2000. 281 pP.

2. Pender - N. 1. Health Promotion in Nursing Practice.Connecticut: Appleton \& Lange. Traducido por: Lic Virginia López. Casados. Minessota, 1996. 25 pp.

3. Lucas - M. S. Estereotipos por razón de género en el autoconocimiento y autoeficacio profesionol. Univerșidad de Valladọlid. Barcelona, 2000. 263 pp.

4. Bandura, A. Self-efficacy mechanism in human agency. American Psychologist, 1982. 384 pp.

5. Contreras - A. Presentación de Modelos Teóricos Conductuales: La Teoría del Aprendizaje. [on line] [citado el 26 de Abril del 2003] Disponible en World Wide Web:

http://www//teoriasdelaprendizaje.htlm.

6. Benitez - T. A. \& Franco - O. M. Factores que intervienen en el conocimiento y aplicación del proceso de enfermería en estudiantes de $4^{\circ}$ semestre de la Licenciatura en Enfermeria (tesis). Escuela Nacional de Enfermería y Obstetricia. Universidad Nacional Autónoma de México. México, 2000. 113 pp.

7. Laschinger - S. K., McWilliam - C. Li, \& Weston - W. El efecto de las rotaciones clinicas de enfermeria familiar y de medicina familiar sobre la autoeficacia de los estudiantes de enfermeria medicina para la asesoria de promoción de salud. Journal of Nursing Education. Vol. 38 (8). E.U. 1999. 347-355 pp.

8. Tresolini y Stritter. En: Laschinger, McWillian y Weston. El efecto de Ias rotaciones alinicas de enfermeria familiar y de medicina farniliar sobre la autoeficacia de los estudiantes de enfermeria medicina para la asesoria de promoción de salud. En la revista: Journal of Nursing Education. Vol. 38 (8). 1994. 191-215 pp.
9. Canto y Rodriguez - J. E Autoeficacia y educación. [on line]. [Citado el 20 de octubre del 2004]. Disponible en World Wide Web de hatp://wwwinaf.cl/heman_saavedra_ 03.htm.

\section{DIRECCIÓN PARA CORRESPONDENCIA:}

Mtra. Martha Lilia Bernal Becerril: marthaliliabb@ hotmail.com Mtra. Gandhy Ponce Gómez: gandhyponce@yah.oo.com.mx. Mtra. Liliana Gonzảlez Juárez: lilil@hotrnail.com.

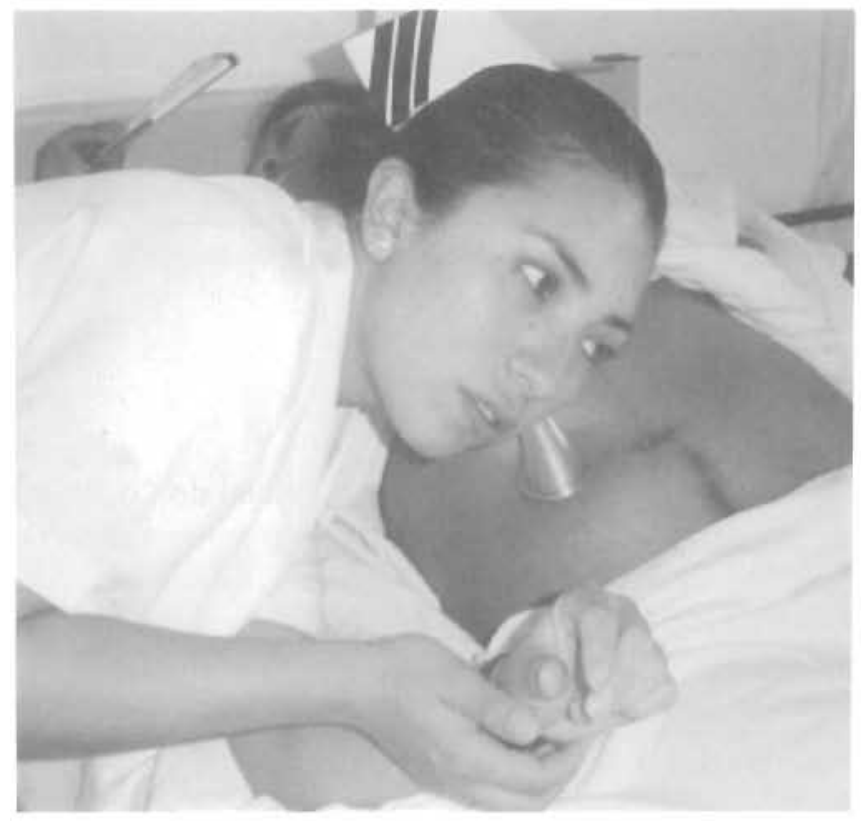

